\title{
Structural Investigation of MOVPE-Grown GaAs on Ge by X-ray
}

\section{Techniques}

C. S. Wong ${ }^{1}$, N. S. Bennett ${ }^{1}$, B. Galiana ${ }^{2}$, P. Tejedor ${ }^{2}$, M. Benedicto ${ }^{2}$, J. M. MolinaAldareguia $^{3}$, P. J. McNally ${ }^{1}$

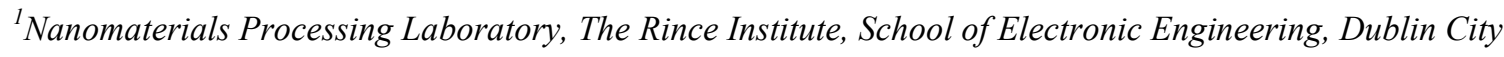
University, Dublin 9, Ireland.

${ }^{2}$ Instituto de Ciencia de Materiales de Madrid, C.S.I.C., Sor Juana Inés de la Cruz 3, 28049 Madrid, Spain. ${ }^{3}$ IMDEA Materials, c/Professor Aranguren s/n, 28040 Madrid, Spain.

The selection of appropriate characterisation methodologies is vital for analysing and comprehending the sources of defects and their influence on the properties of heteroepitaxially grown III-V layers. In this work we investigate the structural properties of GaAs layers grown by Metal-Organic Vapour Phase Epitaxy (MOVPE) on Ge substrates $-(100)$ with $6^{0}$ offset towards $<111>-$ under various growth conditions. Synchrotron X-ray topography (SXRT) is employed to investigate the nature of extended linear defects formed in GaAs epilayers. Other X-ray techniques, such as reciprocal space mapping (RSM) and triple axis $\omega$-scans of $(00 l)$-reflections $(l=2,4,6)$ are used to quantify the degree of relaxation and presence of antiphase domains (APDs) in the GaAs crystals. The surface roughness is found to be closely related to the size of APDs formed at the GaAs/Ge heterointerface, as confirmed by X-ray diffraction (XRD), as well as atomic force microscopy (AFM), and transmission electron microscopy (TEM).

Keywords: GaAs, X-ray diffraction, antiphase domains, dislocations, MOVPE. 


\section{Introduction}

The growth of III-V semiconductor materials on Ge substrates has received enormous research attention due to the potential applications in various microelectronic and optoelectronic devices, such as metal-oxide-semiconductor field-effect transistors (MOSFETS) [1] and multi-junction solar cells [2-3]. A fundamental requirement for monolithic integration of III-V optoelectronics on Ge is the growth of high quality GaAs/Ge films, but the GaAs/Ge material system suffers from inherent issues during growth that are important to overcome. The slight lattice mismatch $(0.1 \%)$ between GaAs and Ge implies that layer relaxation and the generation of misfit dislocations will arise for epilayer growth beyond a certain 'critical thickness'. This however can be resolved by carefully considered pseudomorphic growth [4].

Nonetheless, the growth of polar epilayers (GaAs) on non-polar substrates $(\mathrm{Ge})$ is rather challenging, as it encourages the formation of structural defects such as antiphase domains (APDs), leading to the deterioration of optical properties of the devices fabricated upon it, which is unsuitable for industrial applications [5]. Great strides have been made by various scientists in resolving the problems of APDs appearing in GaAs epilayers on Ge substrates. Successful APD suppression has resulted from the following growth procedures: (i) use of a $6^{\circ}$ offcut Ge substrate to provide a double-step surface [6-8], (ii) forming a single domain surface using an As pre-layer prior to the deposition of a GaAs nucleation layer [910], or (iii) double-step growth - deposition of a GaAs nucleation layer at a lower or higher temperature to that of a GaAs buffer layer [11-13].

The selection of appropriate characterisation techniques is an essential first step to identify significant effects of each growth step (i.e. growth and annealing temperatures, $\mathrm{Ga} /$ As flux ratios and layer thickness), towards production of both APD-free and dislocation- 
free GaAs/Ge films. This study focuses on characterising samples using a range of characterisation methodologies with a particular emphasis on X-ray based techniques, thereby allowing comprehensive information to be extracted. The influences of various growth routines on the strain relaxation process and in suppressing the generation of APDs in GaAs/Ge samples are compared and discussed.

\section{Experimental Details}

Samples have been grown by MOVPE in a 2-inch AIX200/4 horizontal reactor system. The substrates used were p-type Ge (100) wafers, misoriented by 6 degrees towards the nearest (111) plane. The misoriented substrate is vital to produce a double-step surface in order to suppress the formation of APDs at the GaAs/Ge interface [6-8]. The MOVPE growth process took place at a pressure of 100 mbar and with a total flow of 14 slpm of palladiumpurified hydrogen. The precursors used were arsine $\left(\mathrm{AsH}_{3}\right)$ and trimethylgallium (TMGa). A set of GaAs/Ge samples was produced by modifying an optimised routine [14] based on four steps that are consistent with previous work presented in the literature, namely (i) Ge substrate annealing at a high temperature $\left(700^{\circ} \mathrm{C}\right)$, in order to create a double step surface; (ii) an As layer pre-deposition, to achieve a single-domain surface [9-10]; (iii) a GaAs nucleation layer with a thickness of $50 \mathrm{~nm}$ grown at a temperature of $500^{\circ} \mathrm{C}$ [11-13.]; and (iv) a GaAs layer of $600 \mathrm{~nm}$ grown at a higher temperature $\left(640^{\circ} \mathrm{C}\right)$.

Five different samples have been grown and characterised - (1) sample A, grown omitting steps (ii) and (iii), i.e. a GaAs layer grown at $640^{\circ} \mathrm{C}$ just after the Ge substrate annealing at $700^{\circ} \mathrm{C}$ in $\mathrm{H}_{2}$; (2) sample $\mathrm{B}$, grown omitting the low temperature nucleation layer and (3) sample $C$, grown using the full routine. In addition samples D and E were grown in 
the same way as sample B, but in each case a thicker GaAs overlayer was produced (see Figure 1).

These GaAs/Ge samples were first analysed using SXRT to reveal the type and extent of dislocations formed at the $\mathrm{GaAs} / \mathrm{Ge}$ interfaces. The X-ray topographs were taken at HASYLAB-DESY, Hamburg, using the continuous radiation spectrum emitted by a bending magnet source in the DORIS III storage ring. The positron ring at DORIS III had a particle energy of $4.45 \mathrm{GeV}$ and a beam current of 100-150 mA. These topographs were recorded on a high resolution Slavich VRP-M holographic film (grain size $<0.04 \mu \mathrm{m}$ ) set $80 \mathrm{~mm}$ from the sample in back-reflection geometry [15]. LauePT software [16] was used to index the Laue spots in the individual topographs. The topographs were magnified using an optical microscope allowing details of the growth defects to be observed and analysed.

All samples were then examined by a triple-axis Jordan Valley Bede-D1 X-ray diffractometer using a monochromatic $\mathrm{Cu}-\mathrm{K} \alpha_{1}(\lambda=1.5405 \AA)$ radiation source operated at $45 \mathrm{kV}$ and $40 \mathrm{~mA}$. The degree of relaxation of these GaAs/Ge samples was examined using two sets of (224) asymmetric reciprocal space maps (RSMs), recorded by successive 180 degree rotations of the sample around the [001] axis, and the average angular peak separations were used in determining the relaxation degree of the GaAs layer.

In addition to that, these GaAs/Ge samples were further evaluated using triple-axis high-resolution XRD (HRXRD) $\omega$-scans in order to investigate the presence of APDs in GaAs crystal. These measurements were taken at both $(00 l)$-fundamental reflection $(l=4)$ and $(00 l)$-superstructure reflections $(l=2,6)$. The $\omega$-scan full width at half maximum (FWHM) of each sample was extracted and analysed using Williamson-Hall (WM) plots [1719]. The $\beta(\operatorname{Sin} \theta) / \lambda$ factor is plotted against $(\operatorname{Sin} \theta) / \lambda$, where $\beta$ is the FWHM of the $\omega$-scan in angular units, $\lambda$ is the $\mathrm{x}$-ray wavelength, and $2 \theta$ is twice the Bragg angle of the reflection. 
The extracted $\beta$ from $(00 l)$-reflections $(l=2,4,6)$ for each sample is fitted with a linear regression model and the intercept $\left(y_{0}\right)$ of the best-fit straight line corresponding to the lateral coherence length $\left(L_{\|}=0.9\left(2 y_{0}\right)\right)$ of GaAs crystal $[18,19]$. The offcut direction of the sample was placed perpendicular to the direction of the incident beam during the measurements, where the offcut is compensated by optimising the position of $\chi$ on the sample stage to $6^{\circ}$.

In order to monitor how the surface morphology of the GaAs epilayer evolves as the growth condition varies, these GaAs/Ge films were evaluated using AFM. These measurements were carried out using a Digital Instruments-Multimode IIIa microscope working in tapping mode. Si cantilevers (Veeco) with a nominal radius of $10 \mathrm{~nm}$ were used. AFM images with image sizes $5 \mu \mathrm{m}^{2}$ of samples $\mathrm{A}-\mathrm{E}$ were taken. The features of all images were characterised by cross-section profiles and (root-mean-square roughness) $\sigma$ values were calculated and are shown inset in each image.

Additionally, a cross-sectional specimen suitable for high-resolution transmission electron microscopy (TEM) was prepared for samples A, C and E. Dark field and highresolution TEM images were obtained using a Philips CM200 FEG analytical microscope operating at $200 \mathrm{keV}$.

\section{Results and Discussion}

\subsection{Evaluation of the strain relaxation process by SXRT and (224) RSMs.}

Fig. 2 shows the [ $\overline{1} \overline{1} 7]$ large-area back reflection topographs (LABRTs) for GaAs/Ge samples A - E. The projection of the diffraction vector, $\vec{g}$ of the X-ray beam onto the plane of the recording film is represented by the arrow. No images of extended linear dislocations are 
observed in the topograph of sample A (Fig. 2a). Conversely, varying densities of misfit dislocation networks are clearly observed in the topographs of the other samples. From Fig. $2 \mathrm{~b}$, sample B appears to be at an early stage of the strain relaxation process, as only a small number of misfit dislocation networks are observed. This is followed in ascending order by samples C-E as the thickness of the GaAs overlayers increases, while for sample A the GaAs layer is likely to be in near perfect registry with the underlying substrate. Comparing the topograph for each of the samples, the critical thickness $t_{c}$ for misfit dislocation generation is estimated to be $\sim 600 \mathrm{~nm}$, which is higher than the theoretically expected value $\left(t_{c}=300 \mathrm{~nm}\right)$ according to the Matthew-Blakeslee model [20]. A similar phenomenon has been observed by Knuuttila et al. [4], where coherent growth for layer thickness in excess of $t_{c}$ is achievable by carefully selected growth parameters. Although sample $\mathrm{C}$ was grown using different routines compared to that of samples B, D and E, it is also found to be relaxed and contains an intermediate density of misfit dislocations (between B and D). This is thought to be due to the total thickness $(650 \mathrm{~nm})$ of sample $C$ exceeding the $t_{c}$ of $\sim 600 \mathrm{~nm}$ when the additional 50 $\mathrm{nm}$ low temperature GaAs nucleation layer is used prior to the deposition of $600 \mathrm{~nm}$ GaAs buffer layer at $640^{\circ} \mathrm{C}$. Therefore the epilayer is relaxed.

From the dislocation networks observed in a topograph, the dislocation density $\left(\mathrm{cm}^{-2}\right)$ of the particular specimen can be estimated using the following relation [21],

$$
\rho=\frac{L}{V}
$$

where $V$ is the volume of the specimen exposed to the X-rays and $L$ is the total dislocation line length in that volume. $L$ is measured manually from each topograph and volume, $V$, can be easily calculated using the beam spot size and X-ray penetration depth, $t_{p}$. For back 
reflection SXRT, the $t_{p}$ into the sample for each reflection can be calculated based on conventional kinematical theory $[15,22]$

$t_{p}=\left\lfloor\frac{1}{\mu(\lambda)\left(\frac{1}{\sin \phi_{i}}+\frac{1}{\sin \phi_{h}}\right)}\right\rfloor ;$

where $\Phi_{i}$ is the incident grazing angle, $\Phi_{h}$ is the exit grazing angle and $\mu(\lambda)$ is the wavelength-dependent absorption constant for the material. From equations (1) and (2), the dislocation density is then calculated accordingly. This method provides a non-destructive way of estimating dislocation densities throughout the entire film without the need for chemical etching of the layer. Analysis reveals the highest dislocation density $\left(8.9 \pm 0.7 \times 10^{5}\right.$ $\mathrm{cm}^{-2}$ ) in the thickest film - sample E, as presented in Table I. Misfit dislocations are generated in samples B - E, once the thickness of the GaAs epilayer exceeds the experimental critical thickness of $600 \mathrm{~nm}$, where they are created to accommodate the $\sim 0.1 \%$ lattice mismatch between GaAs and the Ge substrate. From the topographs, there is no signature of threading dislocations being observed, and the observed defect images are thought to be contributing mostly from the dislocation networks confined at the regions close to the GaAs/Ge interface.

Turning our attention to non imaging X-ray diffraction measurements, Fig. 3 shows the (224) reciprocal space map (RSM) of sample E, by way of example. When one uses an asymmetric (224) RSM, the lattice parameter information both in the growth axis [001] and the surface direction $<110>$ are simultaneously recorded. The diffracted intensity contours of the RSM are plotted as a function of reciprocal space axes $Q_{x}$ and $Q_{z}$, where $Q_{x}$ and $Q_{z}$ correspond to in-plane and out-of-plane lattice constant wavevectors of the GaAs epilayer, measured in reciprocal space $\left(\AA^{-1}\right)$. Considering the $Q_{x}$ axis of sample E, the GaAs in-plane lattice point is altered with respect to the degree of relaxation (or strain) associated with the 
epilayer (see Fig. 3). The degree of relaxation for samples A - E was calculated using the inplane lattice constant extracted from RSMs through the following relation,

relaxation, $R=\frac{a_{l l}-a_{\text {sub }}}{a_{r}-a_{\text {sub }}} \times 100 \%$

where $a_{\|}, a_{r}$ and $a_{\text {sub }}$ are the GaAs in-plane lattice constant extracted from RSMs, GaAs lattice constant and substrate lattice constant, respectively. The resulting calculated values (see Table I) correlate well with the dislocation densities estimated from SXRT, in that the degree of relaxation and dislocation density increase in parallel as the layer thickness increases.

Concerning the results presented so far, the use of different nucleation conditions (an As pre-deposition monolayer or the additional low temperature $\left(500^{\circ} \mathrm{C}\right)$ deposited $\mathrm{GaAs}$ nucleation layer) do not appear to significantly influence the strain relaxation process. In fact, SXRT and (224) RSMs confirm that the overall GaAs overlayer thickness plays the more important role in determining relaxation, as dislocations start forming beyond the critical thickness, and therefore, increase the dislocation densities of the film (see Table I). Consequently, these results reveal a lack of correlation between the strain relaxation process and the nucleation conditions.

\subsection{The influence of various growth routines on APD self-annihilation in GaAs crystals}

\section{i) Influence of a low temperature deposited GaAs nucleation layer}

Considering samples A-C, growth alterations were employed in order to investigate the effectiveness of growth stages (ii) and (iii) in suppressing the formation of APDs. The Williamson-Hall (WM) plot for GaAs/Ge samples A-C is depicted in Fig. 4. The best straight-line fit was obtained for sample $\mathrm{C}$, with the other samples consistently showing a 
selective broadening characteristic for $(00 l)$-superstructure reflections $(l=2,6)$. This selective broadening is a signature of the presence of APDs $[18,23,24]$ in the GaAs epilayers. This is because the scattering amplitudes of superstructure reflections result from the atomic form factor differences between the long range ordering of $\mathrm{Ga}$ and As atoms in the GaAs crystals $[23,24]$. The existing APDs disrupt the long range periodic arrangement of the $\mathrm{Ga}$ and As atoms where the Ga and As positions are inverted by $180^{\circ}$ with respect to adjacent domains, and lead to additional broadening of the superstructure reflections. Conversely, the scattering amplitudes of the $(00 l)$-fundamental reflection $(l=4)$ arise solely from the sum of atomic form factors of $\mathrm{Ga}$ and As atoms and the crystalline size regardless of whether all lattice sites were randomly occupied by either Ga or As atoms [23, 24], and therefore, this reflection is insensitive to the presence of APDs.

From Fig. 4, the evaluation of the WM plot of sample A shows an average APD domain size $\left(L_{\|}\right)$of $120 \pm 20 \mathrm{~nm}$. The selective broadening of the superstructure reflections confirms the presence of APDs in sample A, due most likely to improper GaAs nucleation routines $[25,26,28,29]$, and thus, the estimated $L_{\|}$corresponds to the average domain size of different APDs appearing in GaAs crystals. As growth stage (ii) was introduced for sample B, the WM plot analysis reveals a drastic reduction in average APD size to $21 \pm 6 \mathrm{~nm}$. A straight line fit of the WM data points was obtained for sample $\mathrm{C}$ when both growth stages (ii) and (iii) were used. This confirms that the sample $\mathrm{C}$ was grown under optimised conditions and is either completely APD-free or the APDs in the GaAs crystal have self-annihilated at an early stage of growth. These XRD results clearly highlight the importance of growth stages (ii) and (iii) for the reduction of the APD size and to a more effective APD self-annihilation routine. Large APDs $(120 \pm 20 \mathrm{~nm})$ are formed at the heterointerface of the sample A due to uncontrolled initial surface nucleation of GaAs epilayer directly onto the Ge substrate [6-10, 
29]. Using just the As pre-deposition (stage ii) does somewhat reduce the domain size by homogenising the Ge surface with As-As dimers to the order of $21 \pm 6 \mathrm{~nm}$ [9-10], yet APDs are still significantly present when the low temperature nucleation layer is omitted (stage iii). The implementation of growth stage (iii) could be responsible for the slow initial nucleation growth that allows atomic rearrangements which effectively annihilate APDs, as a large amount of energy is required to form the boundaries between two adjacent domains [23, 30].

The AFM images for samples A-C are depicted in Figs. 5 a)-c), respectively. A relatively rough surface morphology has been observed at the GaAs surface of sample A, with valleys of up to $120 \mathrm{~nm}$ in depth. The wavy surface morphology is thought to be due to the presence of a high density of APBs in the GaAs layer formed by the co-existence of AsAs and Ga-Ga domains, which has been observed by several authors $[25,26,28]$. The low $\sigma$ value (smoother surface) for sample $\mathrm{C}$ confirms the result of the WM analysis, in that this sample is free or nearly-free of APDs or they are self-annihilated, leading to a better surface morphology [25-27]. In general, the surface roughening of the GaAs buffer layers is highly dependent on the selection of III/V flux ratios, growth temperatures in addition to the presence of APDs [14, 25-28, 29]. By keeping other growth conditions (i.e. flux ratio and growth temperatures) of the GaAs overlayer the same for samples A-C, it would be reasonable to assume that the surface roughening of the GaAs overlayers is most probably related to the presence of APDs in GaAs crystals. Surface roughening can also be due to the strain relief mechanism of the GaAs epilayer, but this effect is relatively small when compared to that of APDs effect for the samples under test. Our results show that most of the strain is relieved through the generation of misfit-dislocations, that are mostly confined at the near-interface region and do not extend towards the top surface. 
The existence of APDs was further verified by cross-sectional transmission electron microscopy (X-TEM). In Fig. 6a), APDs are clearly observed from the contrast discontinuities of the image at the GaAs/Ge interface for sample A and these APDs are confined to a region approximately $200 \mathrm{~nm}$ from the interface with a domain size of around $100 \pm 30 \mathrm{~nm}$ and decrease significantly with distance away from the GaAs/Ge interface. The anti-phase boundaries (APBs) of different domains start inclining with adjacent boundaries, and thus, they are self-annihilated $[6,29,30]$. On the other hand, no significant densities of APDs albeit with the presence of a small number of misfit dislocations are observed at the hetero-interface of sample C. These X-TEM observations are in good agreement with and confirm the aforementioned XRD and AFM discussions. Note, that these APDs are of the order of 21-120 $\mathrm{nm}$ in size, hence they are too small to be resolved by SXRT.

\section{ii) Effect of GaAs epilayer thickness}

In order to observe how the APDs evolve as the GaAs layer thickness increases, we compared the GaAs epilayer thickness running from $600 \mathrm{~nm}$ (sample B) to $800 \mathrm{~nm}$ (sample D), through to $1000 \mathrm{~nm}$ (sample E). The WM plots of samples B, D and E are depicted in Fig. 4. The selective broadening of the superstructure reflections confirmed the presence of APDs in these GaAs films. These observed APDs in samples B, D and E are evaluated to be $\sim 21-23 \pm 6 \mathrm{~nm}$ in size. This is expected as they were grown using identical growth routines except that a thicker GaAs epilayer was produced.

From the AFM analysis, the surface morphology of these samples is relatively rough due most probably to the presence of APDs in the GaAs crystals [5, 25-28], as shown in Figs. $5 \mathrm{~b}), \mathrm{d})$ and e). The $\sigma$ value $(8.2 \mathrm{~nm})$ of the thin-sample $\mathrm{B}(600 \mathrm{~nm})$ is high and follows in 
descending order from sample $\mathrm{D}$ and $\mathrm{E}$ as the thickness of the GaAs overlayer increases to $800 \mathrm{~nm}$ and $1000 \mathrm{~nm}(7.1 \mathrm{~nm}$ and $5.9 \mathrm{~nm})$, respectively. The downward tendency of the $\sigma$ values might suggest a self-annihilation of APDs with thickness $[8,29]$. This is consistent to the observations reported by Li et al. and Hudait et al., in which the wavy morphology caused by APDs is completely suppressed after the growth of a thick GaAs epilayer.

Fig. 6c) shows the cross-sectional TEM image for the $1000 \mathrm{~nm}$ thick sample - sample E, demonstrating crystal defects (both APDs and misfit-dislocations) in the GaAs overlayer. From Fig. 6c), it appears that the APDs are self-annihilated within a short distance from GaAs/Ge interface, which verifies the aforementioned discussions.

Considering now all five GaAs/Ge samples under test, the results from various characterisation techniques suggest that the generation of APDs gives rise to sample surfaces whose roughness depends on the size of APDs formed at the GaAs/Ge interface. By using a low temperature GaAs nucleation layer and/or a high temperature GaAs epilayer, the results show that most of the APDs are annihilated and do not extend to the free-surface. Nonetheless, the formation of large APDs seems to significantly distort the surface morphology of the subsequently grown GaAs buffer layers. The inset of Fig. 5 depicts a clear correlation between these two parameters for samples A-E. Comparing the AFM and XRD results for samples A-E, one can confirm the direct correspondence of the surface roughening to the different size of APDs formed at the GaAs/Ge interface. Therefore, it is important to annihilate the APDs as early as possible during the growth since this results in a relatively small $\sigma$ of $\sim 1.4 \mathrm{~nm}$ (sample C). 


\subsection{Interaction between misfit dislocations and antiphase boundaries}

These findings highlight the importance of using a range of techniques to analyse heteroepitaxial layers where both dislocations and APDs are important considerations. For example, sample A, for which SXRT and (224) RSM measurements show to be dislocationfree and fully matched to Ge substrate, is shown to contain an abundance of large APDs when investigated with $(00 l)$-reflection $(l=2,4,6) \omega$-scans and TEM. Conversely sample B (same thickness), which contains smaller APDs, is found to be at the early stages of the strain relaxation process. This observation implies a strong interaction between APBs and dislocations. Similar observations have been shown by Ringel et al. [32] in the GaAs/Ge material system grown by migration enhanced epitaxy, although in that case the causes were not explained.

This anti-correlation between the density of APDs and the density of dislocations suggests that the APBs of different domains might act to block the formation of misfit dislocations. In general the formation of a dislocation through the strain relaxation process requires the lattice of the GaAs crystal to move by an extra half plane of atoms by the PeierlsNabarro (PN) driving force [33]. According to this model, the magnitude of the PN driving force is closely related to the width of the dislocation $(W)$, which increases as $W$ decreases as given by the following relation [33],

$$
\tau_{P N} \propto G e^{-2 \pi W / b}
$$

where $G$ is the shear modulus and $b$ is the Burgers vector of the dislocation. $W=a / 1-v$, where $a$ is the interplanar spacing and $v$ is the Poisson's ratio. From the PN model analysis, the strong interaction between APBs and dislocations observed in sample A can be explained by a mechanism where APBs are responsible for the cessation of dislocation propagation by 
reducing the width of the dislocation. This is because the bonding forces of As-As or Ga-Ga bonds near to the APBs are highly directional, and therefore the dislocation width is narrow and the Peierls stress is accordingly large $[33,34]$. Nonetheless, as the APDs size reduces from $100 \mathrm{~nm}$ to $25 \mathrm{~nm}$ for sample B, APDs no longer act effectively to block the propagation of misfit dislocations, and therefore, the epilayer starts to relax.

\section{Conclusions}

A range of experimental techniques has been used to investigate GaAs growth on $\mathrm{Ge}$ by MOVPE. The influence of a low temperature GaAs nucleation layer and epilayer thickness on the structural properties of GaAs epilayers has been studied by SXRT, (224) RSMs, triple-axis $\omega$-scans, AFM and X-TEM. In this current sample set, SXRT and (224) RSMs revealed increasing dislocation density and relaxation degree in the thicker GaAs epilayers (samples C, D and E). In contrast, thin (600 nm) films (samples A and B) showed few dislocations and very little relaxation. This result implies a lack of correlation between the dislocation density and the nucleation routine. Conversely, triple-axis $\omega$-scans, AFM and TEM measurements showed that the surface roughness is closely correlated to the APDs size formed in the GaAs crystal. The formation of larger APDs led to rougher surface morphologies. Overall, this work demonstrates the importance of using a series of appropriate characterisation methodologies in identifying the significant effect of each growth process. For example, $(00 l)$-reflection $(l=2,4,6) \omega$-scans showed that APDs are most prevalent in sample A, even though it contained few dislocations and almost no layer relaxation in SXRT and (224) RSM analysis. 
In particular, X-ray techniques can serve as a rapid characterisation methodology in revealing the structural properties of the epilayers. More importantly, X-ray techniques are non-invasive, thereby allowing the same sample to be measured a number of times, allowing comprehensive information to be extracted.

\section{Acknowledgements}

This work was supported by the EU FP7 MNT ERA-Net 'ENGAGE' project with local support from Enterprise Ireland and Fundación Madrimasd para el Conocimiento. Financial support by MICINN under grant TEC2007-66955 is gratefully acknowledged, as are Dr. C. Algora and Dr. I. Rey-Stolle from the Solar Energy Institute of the Polytechnic University of Madrid for access to the MOVPE reactor. PMN also acknowledges the support of the 'INSPIRE' programme, funded by the Irish Government's Programme for Research in Third Level Institutions and Science Foundation Ireland's 'Precision' Strategic Research Cluster (08/SRC/I1411).

\section{References}

1. C. K. Chia, J. R. Dong, D. Z. Chi, A. Sridhara, A. S. W. Wong, M. Suryana, G. K. Dalapati, S. J. Chua, S. J. Lee 2008 Appl. Phys. Lett. 92141905

2. R. R. King et al. 2009 Proceedings of the $24^{\text {th }}$ European Photovoltaic Solar Energy Conference and Exhibition, Hamburg, Germany p 21

3. J. F. Wheeldon et al. 2010 Proceeding of SPIE 7750, Niagara Falls, Canada p $77502 Q$

4. L. Knuuttila, A. Lankinen, J. Likonen, H. Lipsanen, X. Lu, P. Menally, J. Riikonen and T. Tuomi 2005 Jpn. J. Appl. Phys. 447777

5. R. Beeler, J. Mathews, C. Weng, J. Tolle, R. Roucka, A. V. G. Chizmeshya, R. Juday, S. Bagchi, J. Menendez, J. Kouvetakis 2010 Sol. Energy Mater. Sol. Cells 942362

6. L. Lazzarini, L. Nasi, G. Salviati, C. Z. Fregonara, Y. Li, J. J. Giling, C. Hardingham, D. B. Holt 2000 Micron 31217

7. M. K. Hudait, S. B. Krupanidhi 2000 Mater. Res. Bull. 25125 
8. Y. Li, J. J. Giling 1996 J. Cryst. Growth 163203

9. R. Tyagi, M. Singh, M. Thirumavalavan, T. Srinivasan and S. K. Agarwal 2002 J. Electron. Mater. 31234

10. S. Strite, D. Biswas, K. Adomi and H. Morkoc 1990 J. Appl. Phys. 671609

11. J. C. Chen, M. Ladle Ristow, J. I. Cubbage, J. G. Werthen 1992 J. Electron. Mater. 21347

12. M. K. Hudait, P. Modak, S. Hardikar, K. S. R. K. Rao, S. B. Krupanidhi 1998 Mater. Sci. Eng. B 5553

13. S. K. Agarwal, R. Tyagi, M. Singh, R. K. Jain 1999 Sol. Energy Mater. Sol. Cells 5919

14. B. Galiana, K. Volz, I. Rey-Stolle, W. Stolz and C. Algora 2006 Photovoltaic Energy Conversion IEEE $4^{\text {th }}$ World Conference 1807

15. P. J. McNally, R. Rantamaki, T. Tuomi, A. N. Danilewsky, D. Lowney, J. W. Curley, P. A. F. (Tony)

Herbert 2001 IEEE Transactions on Components and Packaging Technologies 2476

16. X. R. Huang 2010 Journal of Applied Crystallography 43926

17. G. K. Williamson and W. H. Hall 1953 Acta. Metall. 122

18. V. K. Dixit, T. Ganguli, T. K. Sharma, S. D. Singh, R. Kumar, S. Porwal, P. Tiwari, A. Ingale, S. M. Oak 2008 J. Cryst. Grwth. 3103428

19. A. K. Rice and K. J. Malloy 2001 J. Appl. Phys. 892816

20. J. W. Matthews, A. E. Blakeslee and S. Mader 1976 Thin Solid Films 33253

21. S. S. Xu and D. Feng 1987 X-ray Diffraction Topography (Science Press, Beijing) p 109

22. G. D. Yao, M. Dudley, and J. Wu 1990 J. X-Ray Sci. Technol. 2195

23. D. A. Neumann, H. Zabel, R. Fischer and H. Morkoc 1987 J. Appl. Phys. 611023

24. L. Kirste, K. M. Pavlov, S. T. Mudie, V. I. Punegov and N. Herres 2005 J. Appl. Cryst. 38183

25. Q. Xu, J. W. P. Hsu, S. M. Ting, E. A. Fitzgerald, R. M. Seig, S. A. Ringel 1998 J. Electron Mater. 271010

26. M. K. Hudait, S. B. Krupanidhi 2000 Materials Research Bulletin 25909

27. I. Nemeth, B. Kunert, W. Stolz, K. Volz 2008 Journal of Crystal Growth 3101595

28. Y. Li, L. Lazzarini, L. J. Giling, G. Salviati 1994 J. Appl. Phys. 765748

29. M. K. Hudait, S. B. Krupanidhi 2001 J. Appl. Phys. 895972

30. O. Rubel, S. D. Baranowskii 2009 Int. J. Mol. Sci. 105104 
31. S. Koh, T. Kondo, T. Ishiwada, H. Sawada, H. Ichinose 2000 Physica E 7876

32. S. A. Ringel, R. M. Sieg, S. M. Ting, E. A. Fitzgerald 1997 Proc $26^{\text {th }}$ IEEE Photovolt. Spec. Conf. p 793

33. R. W. Hertzberg 1996 Deformation and Fracture Mechanics of Engineering Materials, 4th ed. Wiley, New York p 786

34. D. Hull, D. J. Bacon 2007 Introduction to Dislocations, $4^{\text {th }}$ ed. Elsevier, UK p 242 should be 2001 , Oxford, uk 


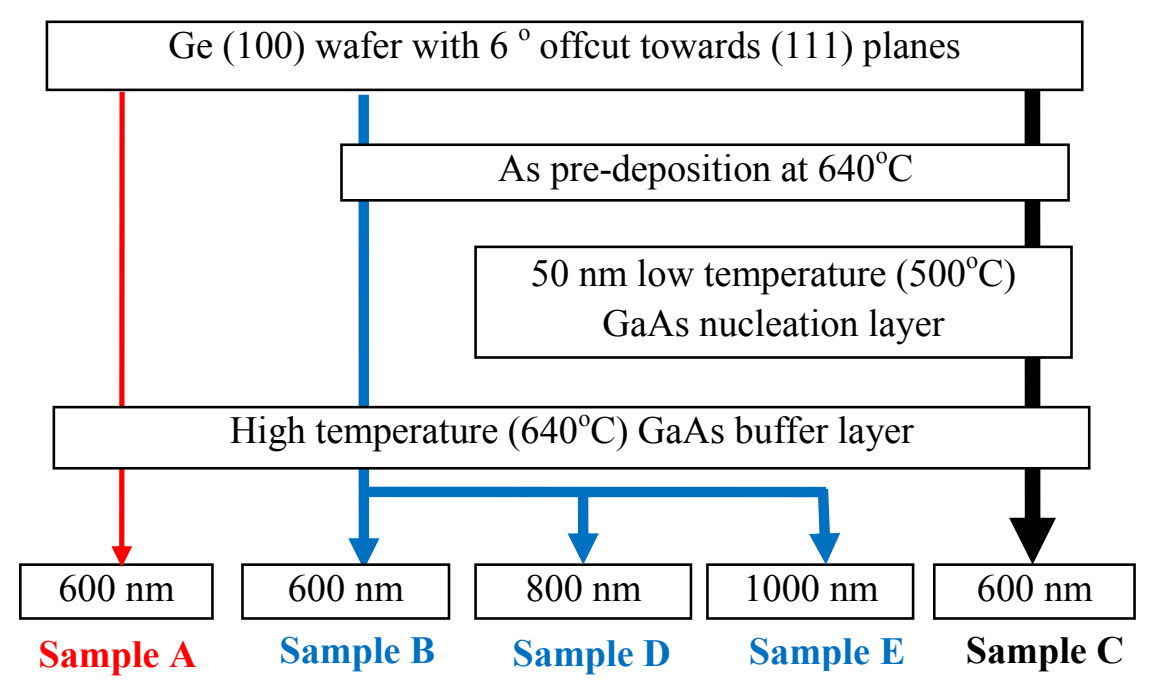

Figure 1. Flow diagram illustrating the growth stages for GaAs/Ge samples A-E, respectively.

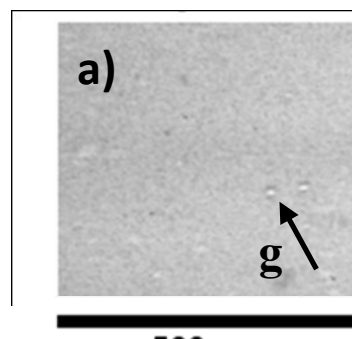

$500 \mu \mathrm{m}$

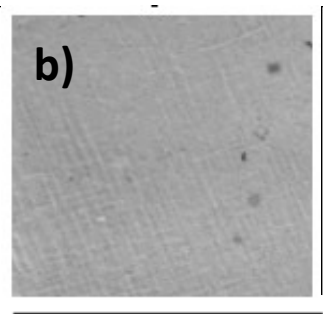

$500 \mu \mathrm{m}$

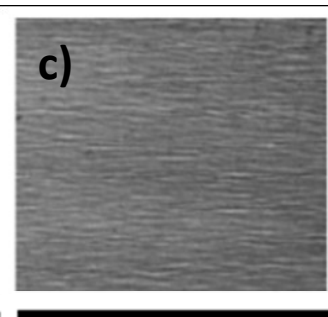

$500 \mu \mathrm{m}$

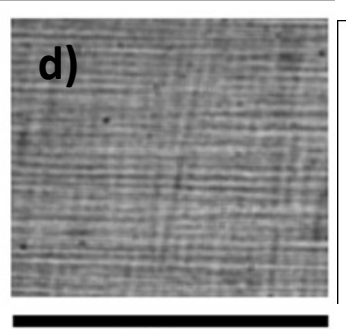

$500 \mu \mathrm{m}$

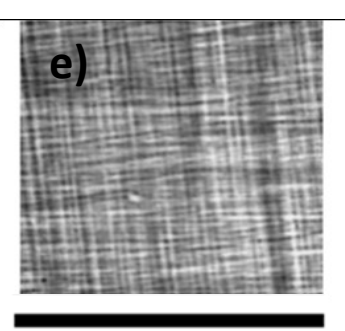

$500 \mu \mathrm{m}$

Figure 2(a) - (e). [ $\overline{\mathbf{1 1}} 7]$ large area back-reflection topographs for GaAs/Ge samples A - E, respectively. The projection of the diffraction vector, $\vec{g}$, for all topographs is shown in (a). 


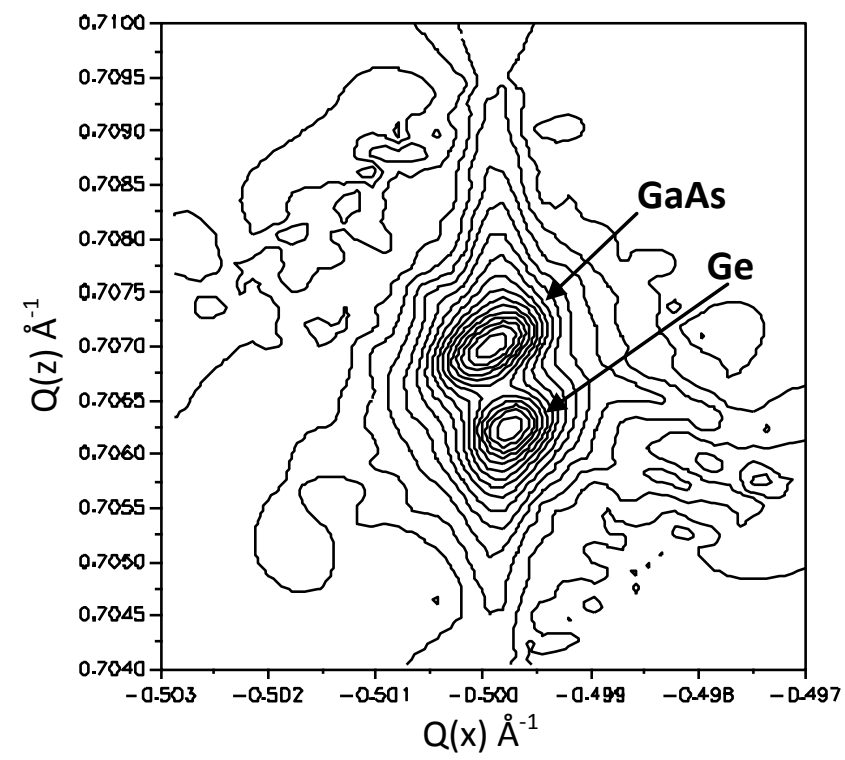

Figure 3. Asymmetric (224) reciprocal space map of sample E.

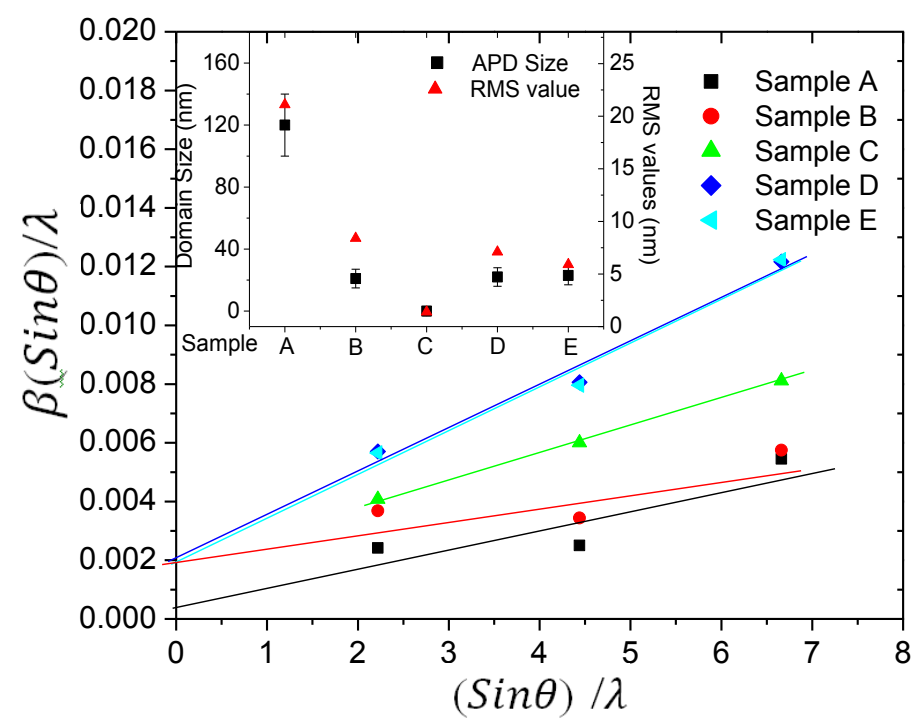

Figure 4. Williamson-Hall plots of GaAs/Ge samples A- E. Inset shows the estimated APD size and RMS value. 

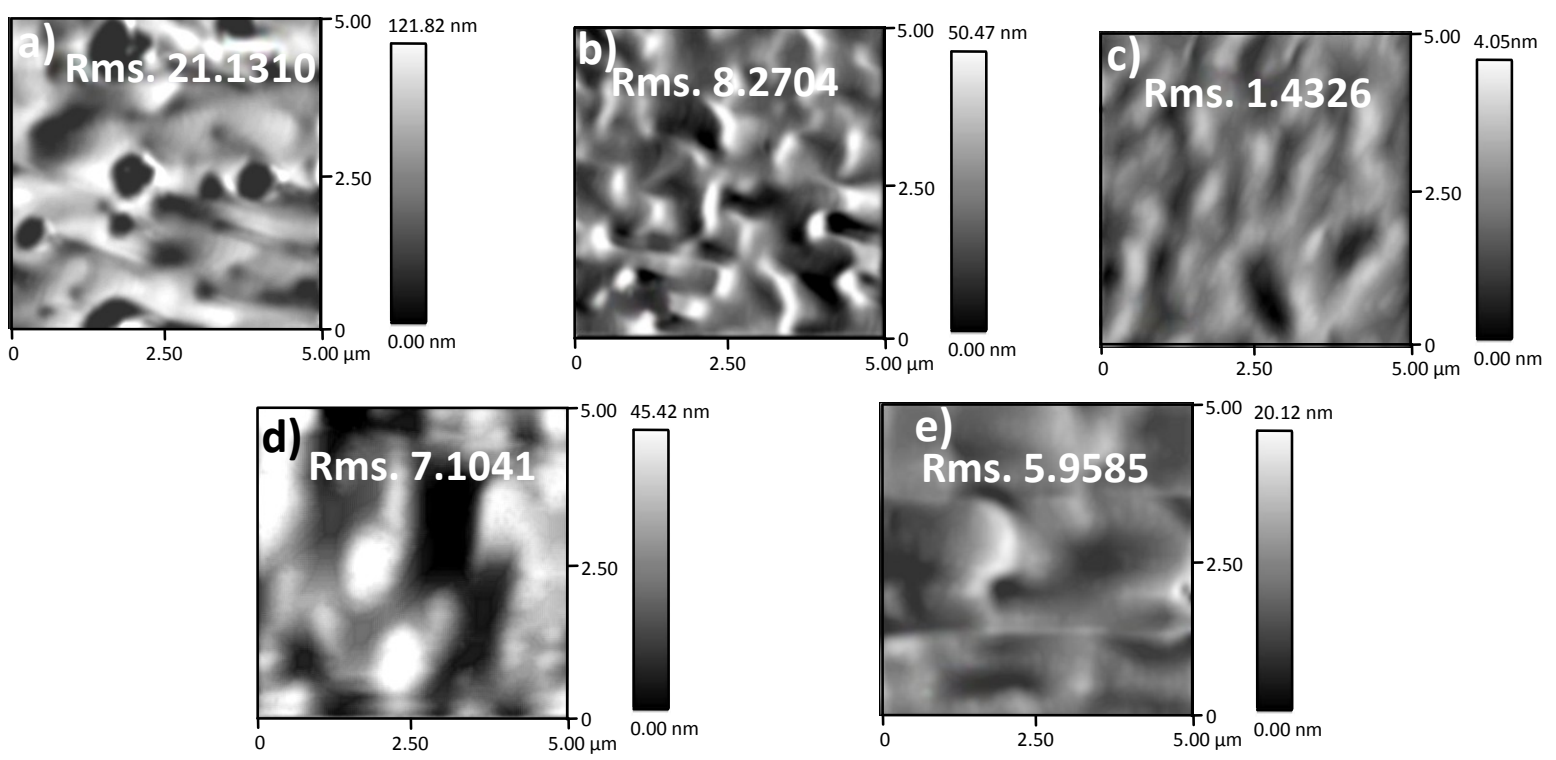

Figure 5 (a) - (e). $5 \mu \mathrm{m}$ x $5 \mu \mathrm{m}$ AFM topographs for GaAs/Ge samples A - E, respectively.
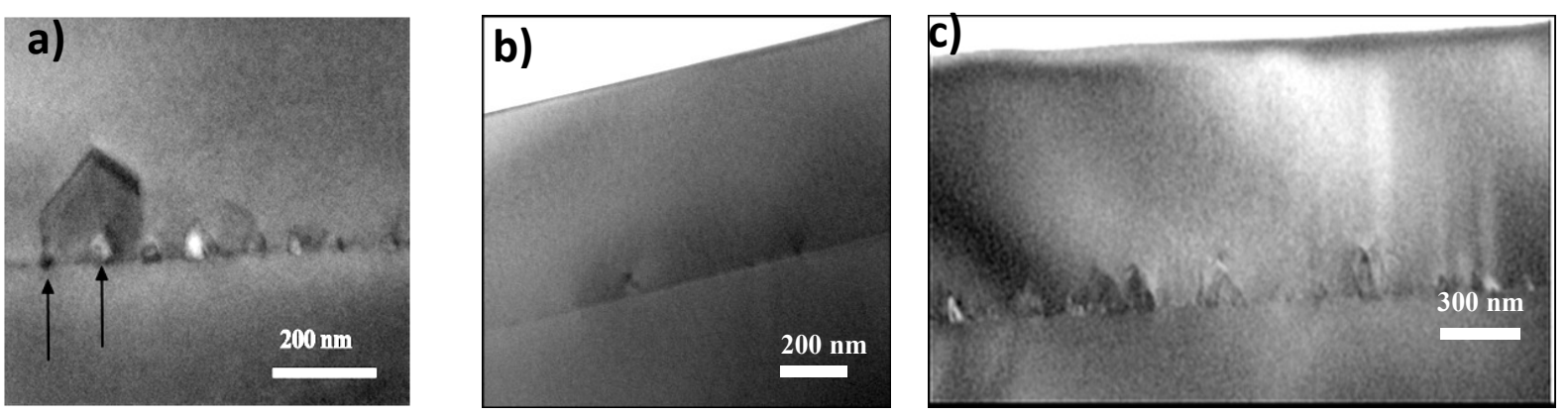

Figure 6 (a) - (c). Cross-sectional transmission electron micrographs of samples A, C and $\mathrm{E}$, respectively, demonstrating crystal defects at the GaAs/Ge interface. 
Table 1 a) Growth parameters, b) dislocation density/ relaxation determined from

SXRT and RSMs, and c) AFM $\sigma$ value and average APDs size for GaAs/Ge samples AE, respectively.

\begin{tabular}{|c|c|c|c|c|c|c|c|}
\hline \multicolumn{3}{|c|}{ a) Growth details } & \multirow[b]{2}{*}{$\begin{array}{l}\text { High temp } \\
\left(640^{\circ} \mathrm{C}\right) \\
\text { GaAs buffer } \\
\text { thickness, } \\
\text { nm }\end{array}$} & \multicolumn{2}{|c|}{ b) $S X R T$ and $R S M$} & \multicolumn{2}{|c|}{ c) $A F M$ and $A P D s$ size } \\
\hline Sample & $\begin{array}{l}\text { As pre- } \\
\text { deposition } \\
\text { at } 640^{\circ} \mathrm{C}\end{array}$ & $\begin{array}{l}\text { Low temp } \\
\left(500^{\circ} \mathrm{C}\right) \\
\text { GaAs } \\
\text { nucleation } \\
\text { thickness, } \\
\text { nm }\end{array}$ & & $\begin{array}{l}\text { Dislocation } \\
\text { density, } \times 10^{5} \\
\mathrm{~cm}^{-2}\end{array}$ & $\begin{array}{l}\text { Degree of } \\
\text { relaxation, } \\
\%\end{array}$ & $\begin{array}{l}\text { Root-mean- } \\
\text { square } \\
\text { roughness, } \\
\text { nm }\end{array}$ & $\begin{array}{l}\text { Average } \\
\text { APDs } \\
\text { size, } n m\end{array}$ \\
\hline A & No & - & 600 & None visible & $<1$ & 21.1 & $120 \pm 20$ \\
\hline B & Yes & - & 600 & $1.2 \pm 0.7$ & $<2$ & 8.2 & $21 \pm 6$ \\
\hline $\mathrm{C}$ & Yes & 50 & 600 & $4.0 \pm 0.7$ & 15 & 1.4 & - \\
\hline $\mathrm{D}$ & Yes & - & 800 & $7.1 \pm 0.7$ & 33 & 7.1 & $22 \pm 6$ \\
\hline $\mathrm{E}$ & Yes & - & 1000 & $8.9 \pm 0.7$ & 48 & 5.9 & $23 \pm 6$ \\
\hline
\end{tabular}

